\title{
Cusp Thickening Severity
}

National Cancer Institute

\section{Source}

National Cancer Institute. Cusp Thickening Severity. NCI Thesaurus. Code C127561.

The qualitative measurement of the severity of a thickening cardiac valve cusp. 